\title{
Complexities surrounding the diagnosis and management of hypercalcaemia in pregnancy
}

\author{
Natassia Rodrigo ${ }^{1,2,3}$, Diana Learoyd $\mathbf{d}^{1,2,3}$ and Sarah J Glastras $1,2,3$ \\ 1Department of Diabetes and Endocrinology, Royal North Shore Hospital, Sydney, Australia, ${ }^{2}$ Northern Clinical \\ School, Department of Medicine, University of Sydney, Australia, and ${ }^{3}$ Kolling Institute of Medical Research, Royal \\ North Shore Hospital, Sydney, Australia
}

Correspondence should be addressed to N Rodrigo Email natassia.rodrigo@sydney. edu.au

\section{Summary}

Hypercalcaemia in pregnancy is uncommon, with associated adverse obstetric and perinatal outcomes for both the mother and the fetus. Determination of causality is central to its management. Diagnostic imaging techniques are limited during pregnancy and the diagnosis is made more complex by physiological changes in calcium and vitamin $D$ homeostasis in pregnancy. Further, therapeutic options are limited due to safety considerations for the pregnant woman and the developing foetus. Three cases of hypercalcaemia in pregnancy will be presented, highlighting the distinct aetiologies and management strategies for hypercalcaemia in pregnancy and the importance of early measurement of serum calcium in pregnancy screening.

\section{Learning points:}

- There are complex physiological changes in calcium balance in pregnancy, including increased calcium intestinal absorption and renal excretion.

- Hypercalcaemia in pregnancy is uncommon but has important potential maternal and foetal complications, making a compelling argument for routine antenatal, calcium screening.

- Identifying the cause of hypercalcaemia in pregnancy can be challenging due to the complex placental interplay in biochemical test interpretation and due to safety constraints restricting imaging and surgery.

- Acute medical management of hypercalcaemia must be considered in the context of both maternal and foetal well-being, along with gestational age and specific consideration for the safety of the developing fetus in late gestation.

\section{Background}

Hypercalcaemia is a rare complication in pregnancy, affecting $0.03 \%$ women of reproductive age (1). It is often asymptomatic making detection difficult, and the most common symptoms are non-specific, such as fatigue and nausea, which can be easily attributed to the pregnant state $(1,2,3)$. Serum calcium is infrequently measured routinely during pregnancy. Therefore, hypercalcaemia in pregnancy is likely underdiagnosed, despite clinically significant implications for morbidity and mortality of both the mother and the fetus. Maternal complications of hypercalcemia include severe hypertension and pre-eclampsia, pancreatitis, nephrolithiasis, and acute kidney injury. Foetal adverse outcomes include intrauterine growth restriction, foetal demise in utero, neonatal hypocalcaemia, tetany and permanent hypoparathyroidism (1). There is likely a gradient of risk depending on the degree of hypercalcaemia, though some case reports have demonstrated adverse fetal outcomes in pregnancies with serum calcium less than $2.85 \mathrm{mmol} / \mathrm{L}(2)$. 
The physiologic changes in calcium and vitamin D homeostasis during pregnancy add complexity to interpretation of diagnostic tests. Interestingly, the foetus exists in a state of relative hypercalcaemia, with serum calcium levels $0.3-0.5 \mathrm{mmol} / \mathrm{L}$ above maternal levels. These higher levels are important for full mineralisation of the fetal skeleton (3). Fetal PTH levels are modulated via fetal calcium sensing receptors, resulting in fetal PTH suppression; maternal PTH does not cross the placenta (1). Fetal calcium homeostasis involves active transport of maternal calcium via the placenta, mediated by PTHrP expression in the placenta, which is upregulated in the third trimester of pregnancy (4). To maintain maternal calcium levels, intestinal absorption of calcium is upregulated, achieved through placental and renal conversion of 25-hydroxyvitamin $\mathrm{D}$ to 1,25-hydroxyvitamin D. Maternal PTH levels fall to the lower end of the normal range in pregnancy, while PTHrP, produced by the placenta, rise (4). Further, pregnancy is associated with an increase in creatinine clearance and glomerular filtration rate, leading to increasing 24-h calcium excretion from the 12th week of gestation (4). Interpretation of biochemical levels, thus, requires contextualisation within pregnancy.

\section{Case presentation 1}

A primigravid 30-year-old Caucasian woman was presented to a district hospital for her first antenatal visit at 33rd weeks of gestation. She reported a known history of primary hyperparathyroidism; laboratory test results from 7 years prior were obtained and showed both elevated corrected calcium $(2.7 \mathrm{mmol} / \mathrm{L}$, normal range (NR): 2.10-2.60 $\mathrm{mmol} / \mathrm{L}$ ), and parathyroid hormone (PTH): $88 \mathrm{nmol} / \mathrm{L}$ (NR: 15-68 mmol/L). She had two previous episodes of uncomplicated nephrolithiasis and 'low BMD' confirmed on prior DEXA bone densitometry. There was no family history of hypercalcaemia. Seven years prior, a parathyroid sestamibi scan failed to localise an adenoma, and the exploratory neck surgery was unsuccessful. At the antenatal visit, she reported symptoms of fatigue and abdominal bloating with confirmed hypercalcaemia (corrected calcium: $3.03 \mathrm{mmol} / \mathrm{L}, \mathrm{NR}: 2.10-2.60$ ).

\section{Investigation case 1}

Repeat laboratory measurements confirmed parathyroid hormone-dependent hypercalcaemia (Table 1).

The 24-h urinary calcium was elevated at $14 \mathrm{mmol}$ per day (NR: 2.5-7.5), however, this result was difficult to

Table 1 Investigations for case 1.

Test

Corrected calcium, $\mathrm{mmol} / \mathrm{L}$

PTH, ng/L

25-hydroxy-vitamin D, nmol/L

\begin{tabular}{ccc}
\hline Value & & Normal range \\
\cline { 1 - 1 } 2.98 & & $2.10-2.60$ \\
115 & & $15-68$ \\
88 & & $50-140$ \\
\hline
\end{tabular}

interpret due to the altered urinary calcium excretion in pregnancy. Neck ultrasound demonstrated the presence of a well-circumscribed ovoid solid lesion measuring $8 \mathrm{~mm}$ by $12 \mathrm{~mm}$ by $7 \mathrm{~mm}$, isoechoic to the thyroid parenchyma and, in the clinical context, a parathyroid adenoma was considered most likely. The provisional diagnosis was primary hyperparathyroidism secondary to a parathyroid adenoma. Due to her pregnant state, a parathyroid sestamibi scan was not performed.

\section{Treatment case 1}

The patient agreed to be admitted to the hospital for the acute management of hypercalcaemia. i.v. normal saline and oral frusemide were commenced to aid calcium excretion. Serum calcium levels declined to 2.7 $\mathrm{mmol} / \mathrm{L}$ within $24 \mathrm{~h}$. Foetal growth scans demonstrated growth consistent with gestational age. Despite medical management, calcium levels rose once again to $2.9 \mathrm{mmol} / \mathrm{L}$ within 3 days. Fetal cardiotocography was performed daily, and no abnormalities were detected. An induction of labour was performed at 39th weeks gestation, resulting in successful vaginal delivery.

\section{Outcome and follow-up case 1}

There were no neonatal complications. The neonate's serum calcium was elevated (3.2 mmol/L, NR: 1.85-2.80) and PTH appropriately suppressed at birth $(<4.0 \mathrm{ng} / \mathrm{L}$, NR: 15-68), however, normalised over the first week of life without intervention. Maternal hypercalcaemia persisted, however, surgical intervention was declined. The mother was advised about potential delayed signs of neonatal tetany.

\section{Case presentation 2}

A 34-year-old woman was presented in her first successful IVF pregnancy to antenatal care at 12 weeks gestation, with asymptomatic mild hypercalcaemia; corrected calcium $2.76 \mathrm{mmol} / \mathrm{L}$, (NR: 2.10-2.60). She had undergone unsuccessful parathyroidectomy 10 years prior, where despite removal of a suspected parathyroid adenoma, 
hypercalcaemia persisted immediately postoperatively. There was no known family history of hypercalcaemia.

\section{Investigation case 2}

The woman was transferred to a tertiary obstetric hospital. PTH-dependent hypercalcaemia was evident (Table 2). In view of her persistent mild hypercalcaemia, lack of complications and unsuccessful definitive treatment, familial hypocalciuric hypercalcaemia (FHH) was suspected. Germline analysis of the calcium sensing receptor gene (CaSR) confirmed a heterozygous missense mutation (variant of heterozygous missense c. $482 \mathrm{~A}>\mathrm{G}, \mathrm{p}$. (Tyr161Cys) in exon 3), thereby, confirming the presence of FHH type 1.

\section{Treatment case 2}

The medical approach involved expectant management. As the CaSR mutation status of the fetus was unknown, an induction of labour was performed in the 37 th week of gestation, resulting in normal vaginal delivery, but with hindsight early delivery was unnecessary.

\section{Outcome and follow-up case 2}

The baby's neonatal calcium levels were measured after delivery and were persistently elevated (3.0-3.2 $\mathrm{mmol} / \mathrm{L}, \quad \mathrm{NR}: 2.13-2.74)$ suggestive of inheritance of the autosomal dominant CaSR mutation. As expected, maternal hypercalcaemia persisted after delivery. She was counselled about the implications for her baby, future pregnancies and for first degree relatives. Genetic testing confirmed FHH type 1 in the child.

\section{Case presentation 3}

A 33-year-old multiparous woman was presented at 29 weeks gestation, with new onset progressive back pain and rapidly deteriorating mobility. MRI of the thoracic and lumbar spine showed multiple lytic lesions. She had a known germline mutation of breast cancer 2 gene (BRCA 2) and had undergone bilateral mastectomy 5 years

Table 2 Investigations for case 2.

\begin{tabular}{|c|c|c|}
\hline Test & Value & Normal range \\
\hline Corrected calcium, mmol/L & 2.62 & $2.1-2.6$ \\
\hline PTH, ng/L & 42 & $15-68$ \\
\hline Urine calcium excretion, $\mathrm{mmol} / \mathrm{day}$ & 8.1 & $2.5-7.5$ \\
\hline
\end{tabular}

previously after detection of a ductal carcinoma in situ. She reported routine annual surveillance with mammogram and ultrasound until pregnancy.

\section{Investigation case 3}

She had moderately severe hypercalcaemia (Table 3), confirming PTH-independent hypercalcaemia. Parathyroid hormone-related peptide (PTHrP) levels were $2.1 \mathrm{pmol} / \mathrm{L}$ (NR: $1.6-6.9 \mathrm{pmol} / \mathrm{L}$ ). Breast and axillary ultrasound demonstrated enlarged axillary lymph nodes and a mass in the left chest wall. Biopsy of the axillary lymph node confirmed invasive ductal carcinoma of the breast, grade 3 (oestrogen and progesterone receptor positive, Herceptin 2 negative). A CTscan of the chest, abdomen and pelvis (with lead shielding of the fetus) demonstrated widespread metastatic disease involving the liver, spine, sternum, ribs, scapulae, pelvis, femora, left mammary node and lymph nodes.

\section{Treatment case 3}

The multidisciplinary team management involved an obstetrician, medical oncologist, endocrinologist, neurosurgeon, acute pain team and social services. Acute management of hypercalcaemia included i.v. frusemide, i.v. normal saline and calcitonin. The corrected serum calcium level declined to $2.8 \mathrm{mmol} / \mathrm{L}$ within 2 days. Fetal cardiotocography monitoring was performed daily and no abnormalities were detected. Tachyphylaxis occurred after 6 days of calcitonin therapy, and the serum calcium level increased to $3.03 \mathrm{mmol} / \mathrm{L}$. Due to symptomatic hypercalcaemia and concern for developing cord compression and pathological fractures, emergency caesarean section was performed at 31 weeks gestation.

\section{Outcome and follow-up case 3}

The premature neonate had normal serum calcium at delivery but required respiratory support in the neonatal intensive care unit. Maternal serum calcium returned to normal hours after birth. PTHrP declined to $<2.0 \mathrm{pmol} / \mathrm{L}$ with normalisation of PTH levels, likely reflecting removal

Table 3 Investigations for case 3.

\begin{tabular}{llccc}
\hline Test & & Value & & Normal range \\
\cline { 1 - 1 } Corrected calcium, mmol/L & & 3.36 & & $2.10-2.60$ \\
PTH, pmol/L & & $<.0$ & \\
25-hydroxyvitamin D, mmol/L & & 75 & $50-140$ \\
\hline
\end{tabular}


of the placental bed and, thus, suggesting placental production of PTHrP. Zoledronic acid was administered intravenously for symptomatic bony metastases and this resulted in asymptomatic transient hypocalcaemia for 3 days (nadir: $2.0 \mathrm{mmol} / \mathrm{L}$ ). In the subsequent weeks, she received surgery to stabilise a pathological hip fracture, spinal radiation therapy, and systemic chemotherapy.

\section{Discussion}

Hypercalcemia in pregnancy is difficult to diagnose clinically because symptoms and signs are vague and may be confused with the physiological changes of normal pregnancy, for example, fatigue and polyuria. There is a compelling argument for routine screening of serum calcium (a non-expensive test) in all pregnant women antenatally when routine blood counts and biochemistry are performed The seriousness of hypercalcaemia in pregnancy necessitates timely diagnosis, institution of therapy and sometimes requires early delivery to circumvent risk. The initial approach to therapy is similar to that for the non-pregnant state, for example, i.v. hydration, but the diagnostic scan and drug treatment and surgery options are limited due to safety concerns.

Primary hyperparathyroidism is the most common cause of hypercalcaemia in pregnancy. Surgery is recommended for primary hyperparathyroidism, best performed in the second trimester (5). Minimally invasive parathyroidectomy is preferred, though four gland neck exploration is required if preoperative investigations fail to localise the adenoma. CT and $99 \mathrm{mTc}$ sestamibi scan are traditionally contraindicated in pregnancy due to radiation exposure risk to the foetus, however, $99 \mathrm{mTc}$ sestamibi has been performed safely in pregnancy in a single tertiary centre, using a 50\% reduction in radiation dose, increased scanning time, and maternal hydration (5). In some instances of mild hypercalcaemia, fluid hydration and close monitoring can suffice until postpartum exploration and surgery can be performed (2).

FHH leads to an elevation in the set point of 'normal' calcium levels. It is a rare autosomal dominant condition (prevalence 1:80,000), with fetal outcomes dependant on the foetal genotype (5). Fetuses that inherit the mutation are similarly asymptomatic. Should the fetus not inherit the mutation, a mismatch then exists between maternal and fetal calcium normal ranges. Fetal exposure to relative hypercalcaemia, thereby, increases its risk of adverse outcomes. Rarely, on inheritance of a compound heterozygous or homozygous mutation in the CaSR, neonatal severe hyperparathyroidism can develop.
This autosomal recessive condition is characterised by severe hypercalcaemia and hyperparathyroidism. There is marked demineralisation and subperiosteal resorption with multiple fractures. This condition is potentially fatal without parathyroidectomy in early life (6).

Hypercalcemia of malignancy in pregnancy is rare. Lytic lesions in bone can lead to increased osteoclastic activity or tumoral PTHrP acting at the PTH receptor, promoting bone resorption and renal calcium absorption (7).

Medical management of hypercalcemia in pregnancy is complex. Calcium lowering strategies are necessarily tempered by the pregnant state as foetal safety is paramount. i.v. fluids constitute the mainstay of therapy, with volume expansion correcting the dehydration associated with hypercalcaemia-induced urinary salt wasting (8). Frusemide is also safe in pregnancy and reduces calcium levels via reduction in the passive reabsorption of cations, facilitating an increase in renal excretion of calcium. Cautious use is needed due to competing volume depletion effects, which have the potential to precipitate in worsening hypercalcaemia $(8,9)$. Calcitonin (pregnancy category B2) decreases hypercalcaemia by inhibiting osteoclast activity, thereby, inhibiting bone resorption and enhancing renal excretion of calcium. It is considered safe in pregnancy as it does not cross the placenta. While its onset of action is in 4-6 h, its efficacy is limited by tachyphylaxis, which can manifest after 48 h (8). Glucocorticoids act to reduce placental and renal production of 1,25-hydroxyvitamin $\mathrm{D}$ and can be used to intercept the increased calcium absorption occurring in pregnancy. Maternal side effects are largely dependent on duration of use.

Medications such as bisphosphonates, which are utilised widely outside of pregnancy to manage hypercalcaemia, cross the placenta and are retained in the skeleton for prolonged periods. Fetal skeletal abnormalities, reduced bone growth and weight and hypocalcaemia at birth have been demonstrated in animal studies. Case reports of women exposed to bisphosphonates have demonstrated mixed results, from no ill effects to congenital malformations. Given these results, its use in pregnancy is relatively contraindicated and is regarded as category B3 (10). Denosumab has been used as a calcium lowering therapy, however, in pregnancy, animal studies have shown increased fetal loss, stillbirths and postnatal mortality. There are no controlled data in human pregnancy $(4,9)$. Cinacalcet (category B3) is a calcimimetic agent which binds to and activates the CaSR, leading to a decrease in PTH secretion. The CaSR 
is present on the placenta, leading to the potential for interruption of placental calcium homeostasis. Though there are no studies, it has the potential to cause fetal hypocalcaemia (1).

In conclusion, hypercalcaemia in pregnancy presents complex diagnostic and management challenges and should be ideally managed in a tertiary centre by a multidisciplinary team including an obstetrician, neonatologist, endocrinologist and sometimes an endocrine surgeon. Routine screening of serum calcium antenatally is recommended due to the non-specific clinical presentation of hypercalcaemia in pregnancy. Diagnostic tests must be interpreted with caution given the complex physiological changes associated with pregnancy. Management strategies centre around mitigating adverse perinatal outcomes. The risk of adverse outcomes for the mother and neonate is significant, and timely delivery in these complex cases needs to be carefully considered.

\section{Declaration of interest}

The authors declare there is no conflict of interest that could be perceived as prejudicing the impartiality of the research reported.

\section{Funding}

This research did not receive any specific grant from any funding agency in the public, commercial or not for profit sector.

\section{Patient consent}

Consent was obtained for the publication of this report.

\section{Author contribution statement}

NR was involved in data collection, manuscript drafting and finalising. DL and SG were involved in intellectual input and performed manuscript editing and review.

\section{Acknowledgements}

The authors thank Associate Professor Roderick Clifton-Bligh who facilitated genetic testing in case 2 and also acknowledge all clinicians involved in the care of the three cases.

\section{References}

1 Rey E, Jacob CE, Koolian M \& Morin F. Hypercalcemia in pregnancy - a multifaceted challenge: case reports and literature review. Clinical Case Reports 20164 1001-1008. (https://doi.org/10.1002/ccr3.646)

2 Hirsch D, Kopel V, Nadler V, Levy S, Toledano Y \& Tsvetov G. Pregnancy outcomes in women with primary hyperparathyroidism. Journal of Clinical Endocrinology and Metabolism 2015100 2115-2122. (https://doi.org/10.1210/jc.2015-1110)

3 Kovacs CS. Calcium, phosphorus, and bone metabolism in the fetus and newborn. Early Human Development 201591 623-628. (https:// doi.org/10.1016/j.earlhumdev.2015.08.007)

4 Kovacs CS \& Kronenberg HM. Maternal-fetal calcium and bone metabolism during pregnancy, puerperium, and lactation. Endocrine Reviews 199718 832-872. (https://doi.org/10.1210/edrv.18.6.0319)

5 McMullen TP, Learoyd DL, Williams DC, Sywak MS, Sidhu SB \& Delbridge LW. Hyperparathyroidism in pregnancy: options for localization and surgical therapy. World Journal of Surgery 201034 1811-1816. (https://doi.org/10.1007/s00268-010-0569-2)

6 Hendy GN, D’Souza-Li L, Yang B, Canaff L \& Cole DE. Mutations of the calcium-sensing receptor (CASR) in familial hypocalciuric hypercalcemia, neonatal severe hyperparathyroidism, and autosomal dominant hypocalcemia. Human Mutation 200016 281-296. (https://doi.org/10.1002/1098-1004(200010)16:4<281::AIDHUMU1>3.0.CO;2-A)

7 Chukir T, Liu Y, Hoffman K, Bilezikian JP \& Farooki A. Calcitriol elevation is associated with a higher risk of refractory hypercalcemia of malignancy in solid tumors. Journal of Clinical Endocrinology and Metabolism 2020105 dgz278. (https://doi.org/10.1210/clinem/dgz278)

8 Patel S, Lyons AR \& Hosking DJ. Drugs used in the treatment of metabolic bone disease. Clinical pharmacology and therapeutic use. Drugs 199346 594-617. (https://doi.org/10.2165/00003495199346040-00003)

9 Green SB \& Pappas AL. Effects of maternal bisphosphonate use on fetal and neonatal outcomes. American Journal of Health-System Pharmacy 201471 2029-2036. (https://doi.org/10.2146/ajhp140041)

10 Okamatsu N, Sakai N, Karakawa A, Kouyama N, Sato Y, Inagaki K, Kiuchi Y, Oguchi K, Negishi-Koga T \& Takami M. Biological effects of anti-RANKL antibody administration in pregnant mice and their newborns. Biochemical and Biophysical Research Communications 2017 491 614-621. (https://doi.org/10.1016/j.bbrc.2017.07.154)

Received in final form 25 March 2021

Accepted 20 April 2021 